\section{Chloroquin-induzierte arzneimitteltoxische Alveolitis}

Zusammenfassung: Das Fallbeispiel zeigt einen 41 Jahre alten Mann mit chronischem diskoiden Lupus erythematodes, der mit Chloroquin behandelt wurde. Zwei Wochen nach Therapiebeginn entwickelte er Dyspnoe, purulentes Sputum, hohes Fieber, diffuse papulöse Hauterscheinungen und diffuse, periphere, homogene Infiltrate im Röntgen-Thorax. Nach Absetzen des Chloroquins kam es zu einer raschen Besserung. Eine später durchgeführte orale Provokation mit Chloroquin zeigte Veränderungen, die auf eine arzneimitteltoxische Alveolitis hinwiesen. In der bronchoalveolären Lavage zeigten sich eine Eosinophilie und ein verminderter CD4/CD8-Quotient. Obwohl zahlreiche Nebenwirkungen einer medikamentösen Therapie mit Chloroquin beschrieben sind, fand sich in der Literatur bislang kein Zusammenhang mit einer akuten Alveolitis.

Chloroquine-Induced Drug-Hypersensitivity: This case history presents a 41-years-old man with chronic discoid erythematosus who was treated with chloroquine. After 2 weeks of treatment he developed dyspnea, purulent sputum, high fever, diffuse papular rash, and diffuse peripheral homogeneous infiltrates on chest x-ray. Rapid recovery occurred after discontinning chloroquine. Subsequent peroral provocation with chloroquine caused symptoms suggestive of hypersensitivity pneumonitis. Analysis of bronchoalveolar lavage fluid showed eosinophilia and a reduced CD4/CD8 ratio. Although treatment with chloroquine is often complicated by side effects, in a literature search we could not find any report on acute pneumonitis.

Key words: Chloroquine - Hypersensitivity pneumonitis

\section{Einleitung}

Bei einem an einer Autoimmunkrankheit leidenden Patienten kann das akute Auftreten von Fieber, Luftnot und pulmonalen Infiltraten durch eine Pneumonie im immunkompromittierten Organismus, die Manifestation der Autoimmunkrankheit oder eine Medikamentenunverträglichkeit bedingt sein [1]. Medikamente, die oftmals zu akuten allergischen Reaktionen führen, sind Penicillin, Sulfonamide, Nitrofurantoin, Goldverbindungen, Penicillamin, Captopril, Carbamazepin, Phenytoin,

Pneumologie 2000; 54: 395-397

(c) Georg Thieme Verlag Stuttgart · New York ISSN 0934-8387
K. Mitja, K. Izidor, E. Mušič

Universitätskrankenhaus für Pneumologie und Allergologie, Golnik/Slovenia

Paraaminosalizylsäure, Imipramin, Chlorpropamid und verschiedene Chemotherapeutika.

Chloroquin ist eine Substanz, die für die Prophylaxe und die Behandlung von Malaria benutzt wird, obwohl die großflächige Resistenz von Plasmodium falciparum den therapeutischen Nutzen zunehmend limitiert. Chloroquin wird ebenfalls für die Behandlung von Amöben-Leberabszessen, photoallergischen Reaktionen und Rheumatischen Krankheiten, wie beispielsweise diskoider und systemischer Lupus erythematodes und Rheumatische Arthritis eingesetzt. Der Einsatz als antirheumatisches Medikament ist durch die Toxizität begrenzt und kommt bei Patienten mit milden Verlaufsformen der Krankheit zum Einsatz. Für Chloroquin sind zahlreiche unerwünschte Wirkungen beschrieben. Kopfschmerzen, Hautausschläge und Juckreiz treten häufig auf. Es kann ebenfalls zu reversibler Keratopathie oder irreversibler toxischer Retinopathie kommen [2].

\section{Fallbericht}

Wir stellen einen 41 Jahre alten Mann mit chronischem Nikotinabusus (20 pack-years) vor. Anamnestisch hatte er einen seit 10 Monaten bestehenden Hautausschlag im Gesicht. Aufgrund der Morphologie stellte ein Dermatologe die Diagnose chronischer diskoider Lupus erythematodes. Es wurde Chloroquin (Resochin ${ }^{\circledR}$ ) in einer Dosierung von $150 \mathrm{mg}$ zweimal täglich über 10 Tage, gefolgt von $150 \mathrm{mg}$ einmal täglich verabreicht. 14 Tage später kam es zur intensivpflichtigen stationären Aufnahme bei thorakalen und abdominellen Schmerzen, Dyspnoe, Benommenheit, purulentem Sputum, Schüttelfrost und Fieber. Zum Aufnahmezeitpunkt lag die Körpertemperatur bei $39^{\circ} \mathrm{C}$. Es bestand ein diffus verteilter papulöser Ausschlag. Auskultatorisch imponierten inspiratorische Rasselgeräusche über der rechten Lunge.

Laborchemisch fanden sich folgende Daten: Blutsenkung $56 \mathrm{~mm} / \mathrm{h}$, C-reaktives Protein $97 \mathrm{mg} / \mathrm{L}$, Gesamtbilirubin $31,1 \mu \mathrm{mol} / \mathrm{L}$, konjugiertes Bilirubin $5,7 \mu \mathrm{l} / \mathrm{L}$, normale Transaminasen, Leukozyten $28,9 \times 10^{9} / \mathrm{L}$ mit $91 \%$ neutrophilen Granulozyten. Die arterielle Blutgasanalyse zeigte ein $\mathrm{pO}_{2}$ von $6,16 \mathrm{kPa}$, ein $\mathrm{pCO}_{2}$ von 3,98 kPa bei Raumluft und ein $\mathrm{PO}_{2}$ von $8,52 \mathrm{kPa}$ bei $100 \%$ Sauerstoff. Das Thorax-Röntgenbild zeigte beidseitige, rechtsbetonte periphere Infiltrate. Im EKG fand sich eine Sinustachykardie mit 120 Schlägen/Minute. Eine Therapie mit Cefpiramide (Tamicin ${ }^{\circledR}$, Lek, Slovenien) und Roxithromycin (Renicin ${ }^{\circledR}$, Lek, Slovenien) wurde begonnen. Die dermatologische Therapie wurde ausgesetzt. In Sputum und Blutkulturen konnte kein Keimnachweis geführt werden. 
Zum Aufnahmezeitpunkt durchgeführte serologische Untersuchungen auf Mykoplasmen, Chlamydien und Legionellen waren negativ. Nach 6 Tagen war der Patient afebril und das Thorax-Röntgenbild weitestgehend normal. Die Gesamtleukozytenzahl lag bei 7,1 × 10\% /L mit 17\% eosinophilen Granulozyten. Der Patient wurde unter der Verdachtsdiagnose auf eine interstitielle Pneumonie entlassen; die serologischen Untersuchungen auf Mykoplasmen, Chlamydien und Legionellen waren einen Monat später weiterhin negativ. Zu diesem Zeitpunkt waren Spirometrie und Thorax-Röntgenbild normal. Die Eosinophilie im peripheren Blut war nicht mehr nachweisbar.

6 Monate später wurde die Therapie mit Chloroquin und zusätzlich Methylprednisolon $8 \mathrm{mg} /$ Tag bei wiederkehrender Dermatitis erneut aufgenommen. 5 Tage nach der ersten Chloroquingabe kam es zu akuter Dyspnoe, Schüttelfrost, Fieber, Arthralgien, Kopfschmerzen und pleuritischen Schmerzen. Bei der körperlichen Untersuchung fanden sich inspiratorische Rasselgeräusche und exspiratorisches Giemen. Das Thorax-Röntgenbild zeigte bilaterale, periphere Infiltrate (Abb.1 a). Die Gesamtleukozytenzahl lag bei $18,3 \times 10^{9} / \mathrm{L}$, das C-reaktive Protein bei $65 \mathrm{mg} / \mathrm{L}$ und der $\mathrm{pO}_{2}$ bei $6,47 \mathrm{kPa}$. Die Spirometrie zum Aufnahmezeitpunkt zeigte eine Reduktion der Vitalkapazität um 50\% mit normaler Einsekundenkapazität. In Sputum- und Urinkulturen konnte kein Keimnachweis geführt werden. Antinukleäre, anti-ds-DNA und antineutrophile Antikörper (ANCA) waren negativ. Der Patient wurde mit Clarithromycin $2 \times 500 \mathrm{mg} / \mathrm{d}$ (Fromilid ${ }^{\circledR}$, Krka, Slovenien) und Methylprednisolon $8 \mathrm{mg} / \mathrm{d}$ behandelt. Nach 7 Tagen waren das Thorax-Röntgenbild und die Spirometrie normal, die Diffusionskapazität lag bei 63\% (Abb.1b). Der Patient wurde unter der Verdachtsdiagnose auf eine chloroquininduzierte Alveolitis entlassen. Es wurde Methylprednisolon $8 \mathrm{mg} / \mathrm{d}$ verschrieben.

Drei Wochen nach der Entlassung wurde der Patient zur medikamentösen Provokation erneut aufgenommen. Er war in guter körperlicher Verfassung, das Thorax-Röntgenbild war normal, die Vitalkapazität lag bei $91 \%$ und die Diffusionskapazität bei $76 \%$. Im peripheren Blut fand sich eine Eosinophilenzahl von weniger als 420 Zellen/ $\mu$ l. In der Bronchoskopie fand sich eine Schleimhaut mit deutlicher Gefäßzeichnung. Die Differenzialzytologie der bronchoalveolären Lavage vor und nach medikamentöser Provokation zeigt Tab.1. Die histologische Aufarbeitung der transbronchialen Biopsien zeigte einen vermutlich durch Zigarettenrauch verursachten chronischen Entzündungsprozess. Als Hinweis auf eine medikamentös verursachte Alveolitis fanden sich Siderophagen, hyperplastische Pneumozyten II und einige eosinophile Granulozyten im Lungenparenchym. 36 Stunden nach Einnahme von $600 \mathrm{mg}$ Resochin ${ }^{\circledR}$ wurde erneut eine bronchoalveoläre Lavage im Mittellappen durchgeführt. Zu diesem Zeitpunkt lag die Zahl der eosinophilen Granulozyten im peripheren Blut bei 1000 Zellen $/ \mu 1$.

Einen Monat später fanden sich im HR-Computertomogramm ein leichtgradig ausgeprägtes Lungenemphysem und ein normales Lungengerüst. Ein Jahr später ist der Zustand des Patienten bei normaler Lungenfunktion stabil.

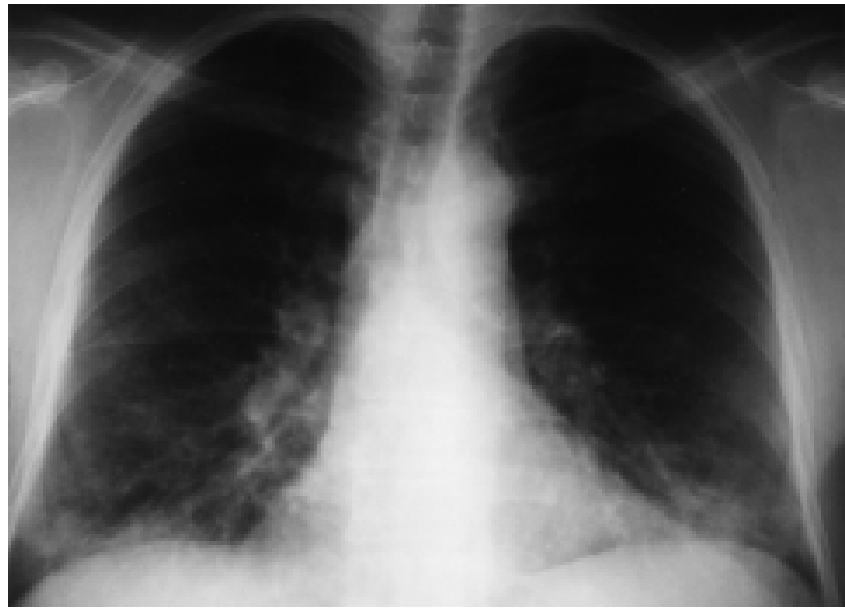

Abb. 1 a Röntgen-Thorax mit bilateralen peripheren Infiltraten fünf Tage nach Beginn des zweiten Therapieintervalls mit Chloroquin bei wiederaufgetretenem diskoiden Lupus erythematodes.

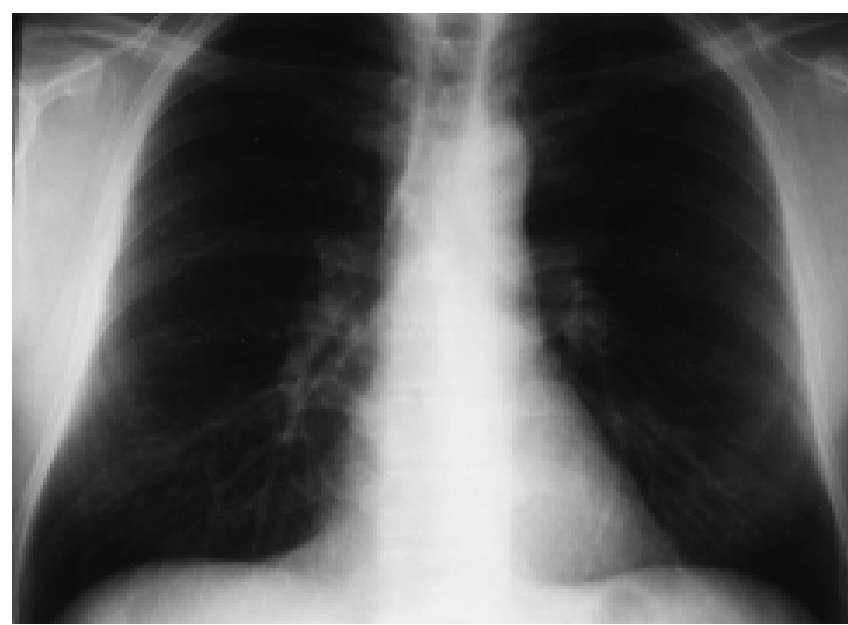

Abb.1b Unauffälliger Röntgen-Thorax sieben Tage nach Absetzen des Chloroquins und Gabe von Methylprednisolon $8 \mathrm{mg} / \mathrm{Tag}$.

Tab. 1 Differenzialzytologie der bronchoalveolären Lavage vor und nach oraler Provokation mit Chloroquin

\begin{tabular}{|c|c|c|}
\hline & vor Provokation & $\begin{array}{l}36 \text { Stunden nach } \\
\text { Provokation }\end{array}$ \\
\hline Zellzahl & 140 Zellen/ $\mu$ l & 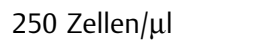 \\
\hline Lymphozyten & 14\% (19,6 Zellen/ul) & 6\% (15 Zellen/ $/ \mu \mathrm{l})$ \\
\hline CD4/CD8 & 0,89 & 0,9 \\
\hline Eosinophile & $3 \%(4,2$ Zellen/ $\mu \mathrm{l})$ & $50 \%$ (125 Zellen/ $\mu \mathrm{l})$ \\
\hline Neutrophile & 2\% (2,8 Zellen/ $\mu \mathrm{l})$ & $<1 \%(<2,5$ Zellen/ $\mu \mathrm{l})$ \\
\hline Basophile & $1 \%(1,4$ Zellen/ $\mu l)$ & $<1 \%(<2,5$ Zellen $/ \mu l)$ \\
\hline Alveolarmakrophagen & $80 \%(112$ Zellen $/ \mu \mathrm{l})$ & 44\% (110 Zellen/ $\mu \mathrm{l})$ \\
\hline
\end{tabular}




\section{Diskussion}

Von einigen Autoren wird die Bezeichnung „exogen-allergische Alveolitis“ ausschließlich für allergische Lungenerkrankungen durch inhalative organische Antigene verwendet. Bei bestimmten medikamentösen Reaktionen finden sich jedoch entsprechende klinische und pathomorphologische Korrelate. Eosinophile Granulozyten sind in der Lunge bei einer großen Anzahl von pulmonalen und systemischen Erkrankungen $\mathrm{zu}$ finden und treten häufig bei medikamentösen Reaktionen auf. Für zahlreiche Medikamente ist eine eosinophile Pneumonie beschrieben, die meistens akut verläuft. Der immunologische Hintergrund ist am besten für Goldsalze untersucht und zeigt als pathogenetische Ursache eine zellvermittelte Immunität. In der bronchoalveolären Lavage zeigt sich eine Lymphozytose mit erniedrigtem CD4/CD8-Quotienten, entsprechend den Befunden bei exogen-allergischer Alveolitis. Die pulmonalen Infiltrate sind häufig von makulopapulösen Hauterscheinungen begleitet. Typischerweise setzt die Krankheit akut ein. Der Verlauf ist normalerweise mild mit Fieber, Husten und Dyspnoe, kann allerdings auch lebensbedrohlich sein. Therapeutisch genügt in der Regel das Auslassen des Medikamentes, eine zusätzliche Gabe von Kortikosteroiden beschleunigt allerdings den Heilungsverlauf. Unser Patient zeigte das typische klinische Bild einer medikamenteninduzierten allergischen Alveolitis.

Differenzialdiagnostisch kann die allergische Alveolitis mit einer infektiösen Pneumonie verwechselt werden, wie es zuerst bei unserem Patienten geschah. Wir vermuteten eine arzneimitteltoxische Alveolitis, nachdem die Krankheit nach wiederholter Gabe von Chloroquin erneut ausbrach. Die Diagnose stützte sich weiterhin auf die Eosinophilie im peripheren Blut, den Hautausschlag und das Verschwinden der pulmonalen Infiltrate nach Absetzen der ChloroquinMedikation. Die postpneumonische Eosinophilie im peripheren Blutbild ist selten ausgeprägt. Die pneumonischen Infiltrate im Thorax-Röntgenbild benötigen in der Regel ebenfalls mehrere Tage bis Wochen für die Rückbildung nach infektiöser Pneumonie.

Da unser Patient an einem diskoiden Lupus erythematodes erkrankt war, vermuteten wir primär eine pulmonale Beteiligung. Da Chloroquin das Standardtherapeutikum des diskoiden Lupus erythematodes ist, musste ein Beweis seiner toxischen Wirkung vor weiterer therapeutischer Gabe geführt werden. Aus diesem Grunde führten wir eine histologische Untersuchung des Lungenparenchyms und eine medikamentöse Provokation mit Chloroquin durch. Die histologische Aufarbeitung der transbronchialen Biopsien zeigte Veränderungen, die mit der Diagnose einer arzneimitteltoxischen Alveolitis vereinbar waren. Die Eosinophilie in der bronchoalveolären Lavage nach Chloroquin-Provokation wies auf eine zellvermittelte Reaktion hin [4].

Obwohl zahlreiche unerwünschte Wirkungen bei der therapeutischen Gabe von Chloroquin bekannt sind, fand sich bislang keine Induktion einer akuten Alveolitis [5].

\section{Literatur}

${ }^{1}$ Mušič E, Tomšič M, Logar D. Goldsalzalveolitis bei drei Patienten mit rheumatoider Arthritis. Pneumologie 1995; 49: 367-372

2 Easterbrook M. Ocular effects and safety of antimalarial agents. Am J Med 1988; 85 (Suppl 4A): 23 - 29

${ }^{3}$ McCormick J, Cole S, Lahiri $\mathrm{C}$ et al. Pneumonitis caused by gold salt therapy: evidence for the role of cell mediated immunity in its pathogenesis. Am Rev Respir Dis 1980; 122: 145-152

${ }^{4}$ Pichler WJ, Zanni M, von Greyerz S et al. High IL-5 production by drug specific T cell clones. Int Arch Appl Allergy Immunol 1997; 113: $177-180$

${ }^{5}$ Jedynak U, Ciezarek M, Kopinski P. Pulmonary infiltrates with masto- and lymphocytosis in BALF associated with arechine. Polish J Pathol 1997; 48: 63-67

\section{K. Mitja}

Universitätskrankenhaus für Pneumologie und Allergologie Golnik 36

4204 Golnik

Slovenia 